\title{
Diagnostic and therapeutic aspects of recurrent renal stone disease
}

\author{
Anthony Meyers, Natalie Whalley and Maria Martins \\ Metabolic Stone Unit, Johannesburg Academic Hospital and Dept. of Medicine, University of the Witwatersrand, \\ Medical School, Johannesburg, South Africa
}

\begin{abstract}
Recurrent renal stones occur in $\pm 7.5 \%$ of Caucasian men and $\pm 3 \%$ of all women. Even with increasing urbanisation, renal calculi are reported in less than $1 \%$ of black South African men and women. Four hundred recurrent stone formers were studied at the metabolic stone clinic using routine and special tests. The appropriate therapy for each subgroup is outlined and studies on various different treatments are presented. In addition, lithogenic risk factors were studied in normal black and white subjects and in black stone formers, in order to clarify the low incidence in the black population.

Patients were classified according to dietary and metabolic lithogenic risk factors. $10 \%$ of stone formers had pure dietary factors. The percentage of stone formers in each of the various metabolic subgroups was as follows: Renal hypercalciuria $12 \%$, Absorptive hypercalciuria 10\%, Mild metabolic hyperoxaluria $20 \%$, Hypocitraturia $50 \%$. Successful therapy in terms of preventing further stone formation was reported using Indapamide, calcium carbonate and potassium citrate in renal hypercalciuria, mild metabolic hyperoxaluria and hypocitraturia respectively. Black volunteer subjects had significantly higher $24 \mathrm{hr}$ urinary sodium excretion and significantly lower $24 \mathrm{hr}$ urinary calcium, ' citrate and cystine excretion than white volunteer subjects. Twenty-four hours urinary values in black stone formers were found to be approaching those levels found in white.
\end{abstract}

\footnotetext{
Correspondence and offprin requests to: A M Mcyers. Chicf Physician. Department of Medicine. Division of Nephrology, 7 York Road. Parktown. 2193. South Africa.
}

As urbanisation occurs in the black population, the incidence of urolithiasis would be expected to increase.

We conclude that a detailed metabolic work-up is essential in the elucidation of the various metabolic risk factors in so-called "idiopathic" $\mathrm{CaOx}$ stone formers. It allows appropriate, specific and highly cost-elfective therapy aimed at the prevention of recurrence.

Key words: calcium oxalate; recurrent stone formers; urolithiasis

\section{Introduction}

Renal calculi occur once in a lifetime in $15 \%$ of all Caucasian men and $6 \%$ of all women and recur in about half of this number. Even with increasing urbanisation, renal calculi are still reported in $<1 \%$ of black South African men and women [1].

Stone formation obeys the laws of thermodynamics. Thus, in the absence of crystal inhibitors, it is only when the concentration of solutes in the urine exceeds the metastable limit, that crystals could aggregate and grow resulting in large agglomerates which are the nuclei of stones [2] (Table 1).

This study deals with the important aspects which determined the epidemiological role of the various promoters and inhibitors in 400 patients with recurrent calcium oxalate $(\mathrm{CaO} x)$ urolithiasis.

Therapeutic strategies used to normalise urinary concentrations so as to prevent further stone formation is also presented. In addition, the various lithogenic risk factors were studied in normal black subjects and black stone formers, in order to clarify 
the reasons of low incidence of kidney stone disease in the black population.

Table 1. Urinary stone promoters and inhibitors

\begin{tabular}{ll}
\hline & Inhibition \\
\hline & \\
Calcium & Magnesium \\
Sodium & Pyrophosphate \\
Oxalate & Citrate \\
Urate & High urine volume \\
Cystine & Tamm-Horsfail protein \\
Low pH & Nephrocalcin \\
Low urine flow & Glycosaminoglycans \\
Tamm-horsfall protein & Crystal matrix protein \\
Bacterial products & \\
\end{tabular}

\section{Methods}

Only patients with recurrent renal calculi were included. Exceptions were made if the patient was from the black population or was young (<20yr). A thorough medical history, including the number of stones passed, was taken. A detailed dietary history with regard to the intake of calcium $(\mathrm{Ca})$, sodium $(\mathrm{Na})$, phosphate, oxalate, protein, magnesium $(\mathrm{Mg})$, potassium $(\mathrm{K})$, carbohydrate and fat was conducted by an experienced dietician. Renal ultrasonography, tomography and excretory urography were done to determine the number and size of residual stones and to exclude medullary sponge kidney.

A full biochemical stone work-up was undertaken. Briefly, a fasting and post $\mathrm{Ig}$ load of calcium, urinary calcium:creatinine ratio was performed as described by Pak to distinguish between the various types of hypercalciuria [3] (Figure 1). Standard laboratory methods were used to measure serum $\mathrm{Ca}$, phosphate, $\mathrm{iCa}$, creatinine, urea, alkaline phosphatase, uric acid, cholesterol, liver function, glucose and full blood count. Serum PTH was measured using an Immulite chemiluminescent enzyme assay (cat. no. LKPHI) and calcitriol was measured using a Nichols Institute radioimmunoassay (cat. no. 40-6090). Two 24hr urines were collected, one on the weekend and one on a weekday. Urinary $\mathrm{Ca}, \mathrm{Na}$, creatinine, urate and phosphate were measured using standard laboratory methods. Urinary citrate and oxalate were measured using Boehringer Mannheim enzyme assay kits (catalogue number 139076 and 755699 repectively). A cyanide-nitroprusside test to exclude homozygous cystinuria was performed on a spot urine specimen [4]. An acid load was performed as described by Wrong and Davies if a 9 am spot urine specimen had a $\mathrm{pH}>5.4$ [5]. Titratable acid and ammonia were determined using standard methods. All available calculi were sent for wet semi-quantitative chemical analysis using a Merckognost kit (cat. no. 1 1003).
More recently, a Coulter Counter Multisizer has been used to measure crystal growth and aggregation [6]. Results obtained from the Coulter were confirmed using $\left[{ }^{14} \mathrm{C}\right]$-oxalate incorporation experiments and scanning electron microscopy [7].

\section{Statistical analysis}

Means, standard deviations, paired and unpaired Ttests were performed using Statgraphics (Statistical Graphics Corporation, Plus*ware).

Fig. 1. Urinary calcium: creatinine ratios in controls, absorptive hypercalciuric (AHC) and renal hypercalciuric ( $\mathrm{RHC}$ ) stone formers, before (B) and after (A) a Ig calcium load.

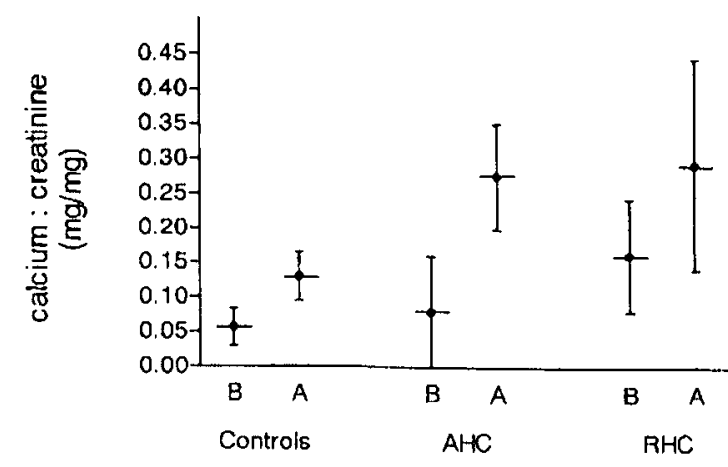

\section{Results}

Lithogenic risk factors and their treament

\section{Dietary factors:}

a) Pure: By definition these patients had only dielary factors without any underlying metabolic abnormality. Dietary aberration as the only risk factor constituted $10 \%$ of 400 patients in the stone population. Within this subgroup, $25 \%$ had high urinary $\mathrm{Na}$ with concomitant high urinary $\mathrm{Ca}$. Once a normal salt diet was attained with a urinary excretion of $<100-120 \mathrm{mmol} /$ day, these patients normalised their urinary calcium (Figure 2 ).

Twenty percent were found to ingest only excessive calcium. Once calcium ingestion, particularly abuse of preparations containing calcium carbonate, was controlled by restriction to $800-1000 \mathrm{mg} /$ day, normocalciuria was attained [8]. It must be stressed that the commonly given advice of severely restricting calcium intake is not only fallacious but dangerous and may lead to, or worsen osteoporosis later on in life. About $20 \%$ had dietary hyperoxaluria and were completely controlled by a low oxalate diet. The main food stuffs containing high quantities of oxalate are Ceylon tea, green leafy vegetables such as spinach and rhubarb, coffee and chocolate. A further $20 \%$ were persistently drinking little fluid resulting in 
low urinary volumes which were highly concentrated. An increased fluid intake controlled these patients. Thus, by correcting the dietary aberrations in this subgroup, stone formation ceased in those who complied.

Fig. 2. Dietary treatment of patients with urinary sodium $>140$ $\mathrm{mmol} / 24 \mathrm{hr}$ with standard sodium $(100 \mathrm{mmol} / 24 \mathrm{hr})$ and calcium (800- $1000 \mathrm{mg} / 24 \mathrm{hr}$ ) diet.

Patients with renal hypercalciuria were excluded from the assessment.

$\mathrm{NMC}=\mathrm{Nil}$ metabolic change. $\mathrm{MC}=$ metabolic change.

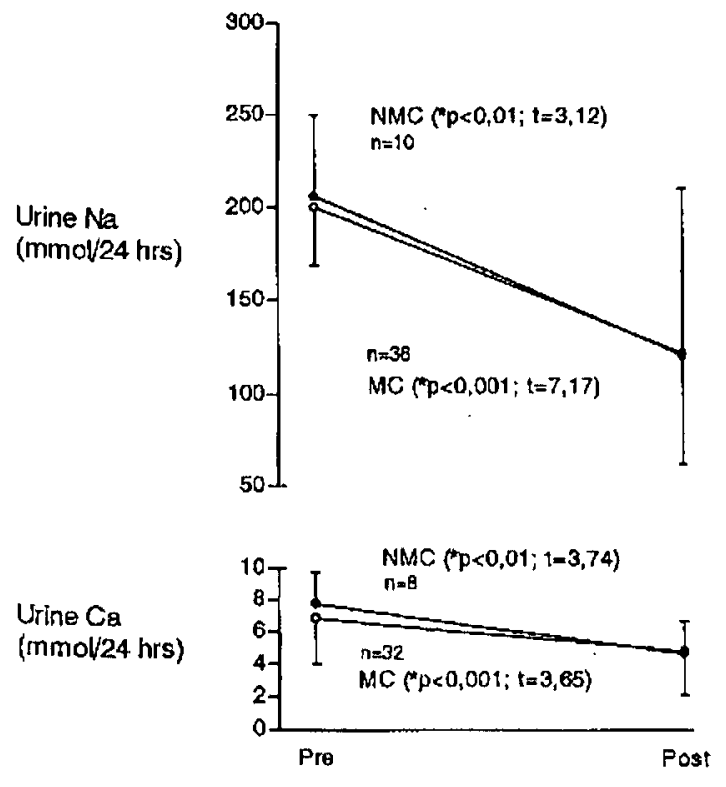

b) Dietary factors in other groups: In all the other metabolic subgroups described below, $40-50 \%$ of the patients had additional dietary aberrations but $50 \%$ did not [8].

Sub-groups according to metabolic risk factors:

1. Hypercalcaemia: Hypercalcaemia secondary to primary hyperparathyroidism, sarcoidosis and various cancers has been noted at our clinic, but this entity, as a cause for renal stones will not be discussed further in this paper.

2. Hypercalciuria: This includes those with absorptive (AHC) and renal hypercalciuria (RHC). Contrary to some reports, our findings agree more with British researchers in that $\mathrm{AHC}$, whilst being a possible risk factor, is only a relatively minor one. In fact, $50 \%$ of these patients do not have hypercalciuria but on calcium:creatinine ratio tests, they were shown to have normal fasting levels but an exaggerated response to a calcium load (Figure 1). Therefore they are probably more at risk to dietary induced hypercalciuria than the normal population. Hypercalciuria as a result of chronic renal calcium loss (RHC), was demonstrated by increased fasting and post $\mathrm{Ca}$ load ratios and was found in about $12 \%$ of all our patients (Figure 1) [8]. The absorption of $\mathrm{Ca}$ from the gut is not disturbed and the cause of the renal loss is unknown. Previous studies have recommended a daily dose of $50 \mathrm{mg}$ of hydrochlorothiazide to normalize hypercalciuria in these patients. Smaller doses are often not potent enough even when combined with ameloride. However, in the doses used, thiazides have significant side-effects eg. hyperlipidemia, hyperuriceamia and carbohydrate intolerance. We have tested the preparation indapamide (Natrilix) which, in a dose of $2.5 \mathrm{mg}$ daily, has little or no natriuretic properties and rarely causes the above side effects. We have shown that it is potently hypocalciuric and completely corrects the renal leak in patients with RHC [9].

Potassium wasting continues to be a minor problem but in the normal population $\mathrm{K}$ does not usually need to be replaced. We strongly recommend that indapamide should be the drug of choice in patients with RHC and recurrent calculi.

3. Mild Metabolic Hyperoxaluria (MMHo) MMHo should not be confused with the condition of hyperoxaluria/ oxalosis. The defect in MMHo is not yet fully understood. We and others have recently theorised that the oxalate digesting colonic bacteria (oxalobacter formigenes) may be deficient in patients with recurrent calcium oxalate calculi [10].

Twenty percent of our patients had MMHo and it is thus one of the most important risk factors for recurrent calculi [8]. Treatment however is problematic. Some mild improvement in some patients was obtained by decreasing oxalate ingestion. We and others have tried small, medium and large doses of pyridoxine but with limited success (Figure 3). More recently the use of calcium carbonate has been investigated and has been found to be successful in some. Calcium so ingested binds to dietary oxalate thus preventing free oxalate from being absorbed in the small and particularly large intestine. The agent of choice is the simple antacid $\mathrm{CaCO}_{3}$ in a dose of $1-2 \mathrm{gm}$ three times a day to be taken with meals (Table 2). Hypercalciuria must be monitored for and when it occurs may occassionally be treated by the addition of Indapamide. In a small group so treated, the drop in urinary oxalate treated has been highly significant (Figure 3 ).

4. Hypocitraturia: Dietary citrate does not influence the citrate appearing in the urine [11]. Citrate is 
filtered in the kidney having been formed as one of the products of the Krebs Cycle and the final urinary concentration depends on the tubular acidbase balance. An intracellular acidosis results in hyperabsorption from the proximal tubule producing hypocitraturia. Citrate protects against stone formation as it is an avid chelator of calcium and also prevents crystal agglomeration [12].

Fig. 3. Assessment of levels of urinary oxalate in patients with hyperoxaluria before and after treatment with calcium carbonate and pyridoxine

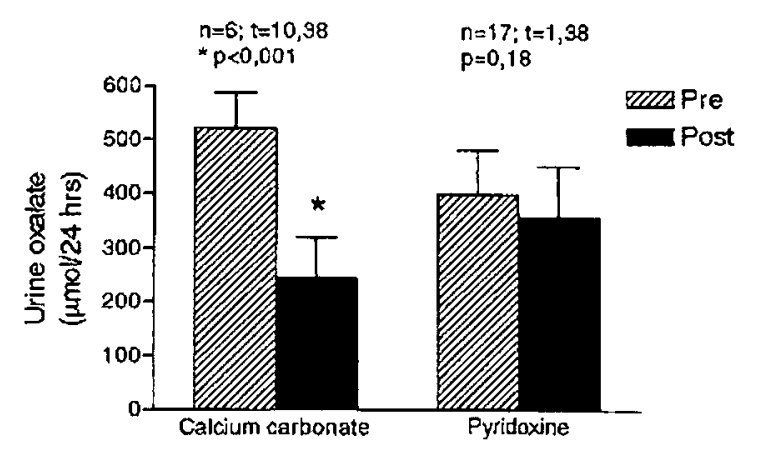

This is the most common inorganic defect found in recurrent calcium oxalate stone formers and occurred in $50 \%$ of all our patients and as an isolated defect in about 15\% [13]. Hypocitraturia is treated by potassium citrate usually in a dose of $15-20 \mathrm{ml}(900 \mathrm{mg} / 5 \mathrm{ml}) 3 \mathrm{x}$ to $4 \mathrm{x}$ a day diluted in a glass of water (Table 2). It is the alkalinising property of this substance that raises urinary citrate and not the citrate itself. Potassium citrate and not sodium citrate is our agent of choice as an increase in the sodium load may result in hypernatriuria with concomitant hypercalciuria and may also possibly worsen hypertension. Potassium citrate therapy decreased stone formation dramatically in a group of 27 patients studied over a 3-7 yr. period [14].

5. Cystine: This is a highly insoluble amino acid and cystinuria is inherited in a complex autosomal recessive fashion. Homozygous individuals form many stones. Cystine is only soluble in large volumes or in urine with a $\mathrm{pH}>7.4$ [15]. It has been noted that several patients who formed calcium oxalate stones also had cystine present in the stones. Of these, $50 \%$ were found to have urinary cystine in the heterozygous range. In addition, in six stones analysed from homozygous cystinurics, 4 were $\mathrm{CaOx}$ mixed with cystine and 2 were pure $\mathrm{CaOx}$. This prompted a study using the Coulter multisizer to examine the effects of varying concentrations (80-320umol/l) of cystine on $\mathrm{CaOx}$ precipitation, growth and aggregation. The metastable limit of the urine was not altered by the increasing cystine concentrations although increased precipitation of $\mathrm{CaOx}$ occurred [16]. Thus epitaxy is unlikely to be the mechanism involved in the $\mathrm{CaOx}$ precipitation by cystine. In addition, the increasing concentrations of cystine resulted in increased growth and aggregation of $\mathrm{CaOx}$. It would appear as though cystine is "salting out" the CaOx as no cystine crystals were noted on light or scanning electron microscopy [16]. Thus cystine may be an important risk factor in the formation of $\mathrm{CaOx}$ stones in heterozygous cystinurics as well as in patients with low urine volumes and excreting concentrated urine. Treatment entails alkalinisation of the urine, probably with potassium citrate, and a high fluid intake ('Table 2).

6. Uric acid: Pure uric acid stones are rarely seen in temperate climates today (only one third is associated with gout). However uric acid associated with calcium oxalate stones is frequently seen. Hyperuricosuria may be due to an increased ingestion of red meat. More commonly, uric acid in the stone is associated with normal urinary uric acid concentration. However uric acid has been shown to 'salt out' calcium oxalate especially in acidic urine [17]. Therefore, the treatment of choice would be to alkalinise the urine again using potassium citrate. If an alkaline urine is attained and maintained the ability of urate to salt out calcium oxalate would be inhibited. Whether the combination of Allopurinol and potassium citrate is more cffective remains to be tested (Table 2 ).

Table 2. Lithogenic risk factors and their treatment

\begin{tabular}{|c|c|}
\hline Risk facior & Treamem \\
\hline Hypercalciuria -Dietary $\mathrm{Na}$ & Sodium Diet $<100 \mathrm{mmol} /$ day \\
\hline Hypercalciuria-Dietary Ca & Calcium Diet $800-1000 \mathrm{mg} /$ day \\
\hline Dietary Hyperoxaluria & Low Oxalate Diet \\
\hline Low Urine Volume & Increase Fluid intake \\
\hline Hypercalciuria- AHC & $\begin{array}{l}\text { Calcium } 800-1000 \mathrm{mg} / \mathrm{day}, \\
\text { fluid }\end{array}$ \\
\hline Hypercalciuria- RHC & Indapamide $2.5 \mathrm{mg} /$ day \\
\hline MMHo & $\begin{array}{l}\text { Ca Carbonate } 1-2 \mathrm{gm}, 2-3 x \\
\text { daily with meals Pyridoxine }\end{array}$ \\
\hline "4 & $25 \mathrm{mg}$ lid \\
\hline Hypocitraturia & $\begin{array}{l}\text { K Citrate } 15-20 \mathrm{ml}(900 \mathrm{mg} / \\
5 \mathrm{ml}) 3-4 \times \text { daily }\end{array}$ \\
\hline Cystine & Fluid, K Citrate \\
\hline Urate in slones & K Citrate and Allopurinol \\
\hline
\end{tabular}


Other important organic inhibitors

Crystal matrix protein:

This protein has been isolated and sequenced and found to be the Fl fragment of prothrombin [18]. Using immunohistochemical methods it has been shown that this protein exists in high concentrations in proximal tubules, distal tubules and the zona glomerulosa of the distal convoluted tubule [19]. Its formation in the kidney is still to be proved using mRNA probes. In Coulter experiments, it was shown to be one of the most potent organic inhibitors of $\mathrm{CaOx}$ crystal growth and aggregation [20].

Much work needs to be done to determine the importance of this inhibitor in normal black and white subjects as well as patients with recurrent stone disease.

Glycosaminoglycans and Tamm-Horsfall protein:

The inhibitor or promoter function of these organic substances need to be further elucidated.

Conflicting reports of both inhibition and promotion of $\mathrm{CaOx}$ formation have been published [7, 21]. It is our intention to examine these substances in the urine of normal blacks and whites using an ELISA assay to determine if any differences are to be found.

Studies in black and while subjects and black and white stone formers

Black and white controls:

Thirty-one semi-urbanised black and 29 urbanised white subjects were studied using methods described above.

Black controls diet was significantly higher in sodium content $(p<0.04)$. There was no difference in serum results.
Urinary sodium was significantly higher $(p<0.0001)$ in black controls than in whites.

Urinary potassium, calcium, citrate, phosphate and cystine were all significantly lower in black controls than in whites; $\mathrm{p}<0.001, \mathrm{p}<0.0001, \mathrm{p}<0.0001$, $\mathrm{p}<0.0005$ and $\mathrm{p}<0.03$ respectively (Table 3 ).

Table 3. Twenty-four hour urine biochemistry in normal black and white subjects.

\begin{tabular}{lll}
\hline & $\begin{array}{l}\text { Black } \\
(n=31)\end{array}$ & $\begin{array}{l}\text { White } \\
(n=29)\end{array}$ \\
\hline Volume ml & $1132(413)$ & $1302(444)$ \\
Creatinine mmol & $12.0(3.1)$ & $12.8(3.9)$ \\
Sodium mmol & $173(63)^{* * *}$ & $113(40)$ \\
Potassium mmol & $45.5(16.2)^{* * *}$ & $73.4(22.0)$ \\
Calcium mmol & $1.53(1.1)^{* * *}$ & $3.51(2.04)$ \\
Phosphate mmol & $18.0(5.7)^{* *}$ & $27.3(12.2)$ \\
Urate mmol & $1.48(0.8)$ & $1.58(0.95)$ \\
Oxalate umol & $272(197)$ & $236(132)$ \\
Cystine mg & $44.4(21.4)$ & $71.7(37.6)$ \\
Citrate mmol & $1.58(0.95)^{* * *}$ & $1.39(0.92)$ \\
\hline
\end{tabular}

Mean (SD) is given. Unpaired Ttest $p<0.03 *, p<0.001 * *$, $\mathrm{p}<0.0001^{* * *}$

Certain intrinsic factors in the South African black may account for the discrepancy in stone formation. Of these, the very low urinary calcium, significantly lower urinary cystine and different interactions between sodium and calcium/cystine are probably of importance [22].

Black and white stone formers:

Twelve healthy volunteer black males (BC), 2 I black stone formers (BSF) and 122 white recurrent stone formers (WSF) were compared. A standard metabolic work-up as given in methods was followed.

Table 4. 24hr urinc results in BC, BSF and WSF

\begin{tabular}{llll}
\hline & $\begin{array}{l}B C \\
(n=12)\end{array}$ & $\begin{array}{l}B S F \\
(n=21)\end{array}$ & $\begin{array}{l}W S F \\
(n=/ 22)\end{array}$ \\
\hline $\mathrm{Vol}(\mathrm{ml})$ & $1041(361)^{*}$ & $1630(717)$ & $1553(607)^{* *}$ \\
$\mathrm{Na}(\mathrm{mmol})$ & $153(64)$ & $134(58)^{*}$ & $164(54)$ \\
$\mathrm{Ca}(\mathrm{mmol})$ & $0.96(0.52)^{* * *}$ & $3.67(2.06)^{* * *}$ & $5.84(2.7)^{* * * *}$ \\
Creat $(\mathrm{mmol})$ & $\mathrm{w} 3.63(1.8)$ & $14.2(4.0)$ & $16.0(5.3)^{*}$ \\
Phos (mmol) & $12.5(3.8)$ & $24.3(13.5)^{* *}$ & $34.9(14.2)^{* * * *}$ \\
Urate $(\mathrm{mmol})$ & $16.9(4.6$ & $1.77(.98)$ & $2.00(1.08)^{* *}$ \\
Citrate $(\mathrm{mmol})$ & $1.06(.72)^{*}$ & $1.04(0.71)^{* * *}$ & $2.06(1.14)^{*}$ \\
& $1.22(1.20)$ & & $318(140)$ \\
Oxalate (umol) & $\mathrm{w} 3.39(1.1)$ & $309(154)$ & $\mathrm{r}=0.40$ \\
Na:Ca & $239(135)$ & $\mathrm{r}=0.45$, & $\mathrm{p}<0.0001$ \\
& $\mathrm{r}=0.23$ & $\mathrm{p}<0.04$ & \\
\hline
\end{tabular}

Results expressed as mean (SD). Ttest $p<0.04^{*}, p<0.01^{* *}, p<0.001^{* * *}, p<0.0001^{* * * *}$. NS $=$ not significant. $w=$ white controls. 
There were no differences in serum calcium, $\mathrm{iCa}$, and phosphate. However the PTH and calcitriol levels were significantly greater in black stone formers as compared with white stone formers. Serum urate and creatinine increased in the black stone formers to a level similar to that found in white stone formers but higher than the black volunteers. Urinary calcium, creatinine, phosphate and urate gave values WSF $>$ $\mathrm{BSF}>\mathrm{BC}$. Urinary citrate was lowest in the BSF < $\mathrm{BC}<\mathrm{WSF}$. Urinary volumes were greater in the stone formers and urinary oxalate was similar in black and white stone formers, both being greater than values found in black volunteers (Table 4).

The various categories of risk factors as found in the black and white stone formers were as follows: Nil metabolic change in $10 \%$ Vs $5 \%$. Metabolic factors $(\mathrm{B}=16$ and $\mathrm{W}=87)$ including: Renal hypercalciuria $4.5 \%$ Vs $10 \%$, absorptive hypercalciuria $4.5 \%$ Vs $12 \%$, MMHo $9 \%$ Vs $26 \%$, Hypocitraturia $82 \%$ Vs $52 \%$.

Pure dietary factors $(B=5$ and $W=35)$, increased urinary calcium as a result of increased urinary sodium $20 \%$ Vs $28 \%$, low volume $17 \%$ Vs $14 \%$. Dietary hyperoxaluria and mixed dietary factors were similar.

It was observed that MMHo was three times more, RHC and AHC were twice as common in WSF. Urinary hypocitraturia is the single most common defect in both groups.

As urbanization occurs, the urine of black stone formers is becoming more like that of the white stone formers. Predictably nephrolithiasis will become more common in the black population [23].

In conclusion, in correctly diagnosed and compliant patients, stone formation can be significantly prevented. Thus, although the metabolic work-up is costly, it is still far more cost effective than repetitive urological procedures. This concurs with the conclusions reached by Parks and Coe [24].

\section{References}

1. Pantanowitz D, Pollen J J, Politzer W M, et al. Urinary calculi S. Afr. J. Med. 1973; 43:128

2. Rao P N, Kavanagh J P, Tiselius H-G. Urolithiasis. Concensus and controversies. Rao P N and Kavanagh, Manchester, UK, 1995; 101-109, 111-117.

3. Pak C Y C, Kaplan R, Bone H et al. A simple test for the diagnosis of absorptive, resorptive and renal hypercalciuria. N. Engl. J. Med. 1975; 292: 497-500.

4. Tietz N W: Textbook of clinical chemistry. W B Saunders Company, Philadelphia, 1986; 556.

5. Wrong O, Davies H E.F. The excretion of acid in renal disease. Q. J. Med. 1959; 28: 259-313.

\section{Acknowledgements}

Many of the studies mentioned in this paper were supported by grants from the South African Medical
6. Ryall R L. Hibbard C M, Marshall V R. A method for studying inhibitory activity in whole urine. Urol. Res. 1985; $13: 285-289$.

7. Grover P K, Ryall R L, Marshall V R. Does Tamm-horsfall mucoprotein inhibit or promote calcium oxalate crystallization in human urine? Clin. Chim. Acta. 1990; 190: 223-238.

8. Gellman L. Recurrent idiopathic calcium urolithiasis at the Johannesburg hospital metabolic stone clinic.1996; MMed thesis, University of the Witwatersrand.

9. Martins M C, Meyers A M, Whalley N A. et al. Indapamide (natrilex): the agent of choice in the treatment of recurrent renal calculi associated with idiopathic hypercalciuria. $\mathrm{Br}$. J. Urol. 1996; $78: 176-180$.

10. Allison M J, Dawson K A. Mayberry W R et al. Oxalobacter formigenes gen. Nov., sp. nov: Oxalate-degrading anaerobes that inhibit the gastrointestinal tract. Arch Microbiol 1985; 141:1-7.

11. Ryall R, Bais K, Rofe A M et al. Urolithiasis 1994; 2. Plenum Press, New York 427.

12. Hamm L L. Renal handling of citrate. Kidney Int. 1990; 38: 728-735.

13. Laminski N A. Meyers A M, Sonnekus M I et al. Prevalence of hypocitraturia and hypopyrophosphaturia in recurrent calcium stone formers: as isolated defect or associaled with other melabolic abnormalities. Nephron 1990: 56: 379-386.

14. Whalley N A, Meyers A M, Martins M C el al. Long term effects of potassium citrate therapy on the formation of new stones in groups of recurrent stone formers with hypocitraturia. Br. J. Urol. 1996; $78: 10-14$

15. Stanburg J B, Wyngaarden J-B, Fredrickson D J et al. The metabolic basis of inherited disease. McGraw - Hill Book Company 1983; 1774-1791.

16. Martins $M$, Whalley N A, Meyers A M. Cystine: Risk factor in the precipitation of calcium oxalate calculi. (abstract) Afran Congress Sept. 1997, Durban - South Africa.

17. Grover P K, Ryall R L, Marshall V R. Effect of urate on calcium oxalate crystallization in human urine: evidence for a promotory role of hyperuricosuria in urolithiasis. Clin. Sci. 1990; 79: 9-15.

18. Stapleton A M F, Ryall R L. Blood coagulation proteins and urolithiasis are linked: crystal matrix protein is the FI activation peptide of human prothrombin. $\mathrm{Br}$. J. Urol. 1995; 75: $712-719$.

19. Stapleion A M F, Scymour A E, Brennan J $S$ et al. Immunohistochemical distribution and purification of crystal matrix protein. Kidney Int. 1993; 44 :817-824.

20. Rao P N, Kavanagh J P, Tiselius H-G. Urolithiasis: Consensus and controversies. Rao P N and Kavanagh J P, Manchester. UK, 1995; 281-283.

21. Hesse A, Wuzel H, Vahlensieck W. The excretion of glycosaminoglycans in the urine of calcium-oxalate-stone patients and healthy persons. Urol. int. 1986; $41: 81-87$.

22. Laminski $N$ A, Moraes $M$, Kruger $M$ et al. Chemical composition of urine in the normal population. A comparison of blacks (B) and whites (W). (abstract) Kidney Int. 1989; 35 : 912.

23. Whalley $N$ A, Martins $M$, Sonnekus $M$ et al. Lithogenic factors in black controls and black and white recurrent stone formers. (abstract) Afran Congress, Sept. 1997. Durban South Afrca.

24. Parks J H, Coe F L. The financial effects of kidney stone prevention. Kidney Int. 1996; $50: 1706-1712$.

Research C̈ouncil and National Kidney Foundation of South Africa. Thanks to Dr Christine Gaillard for translating the abstract into French and to Beverley Smith for helping type the manuscript. 